\title{
Acoustics of monodisperse open-cell foam: An experimental and numerical parametric study
}

\author{
V. Langlois, ${ }^{1, a)}$ A. Kaddami, ${ }^{1}$ O. Pitois, ${ }^{1}$ and C. Perrot ${ }^{2, b)}$ \\ ${ }^{1}$ Lab Navier, Univ Gustave Eiffel, ENPC, CNRS, F-77447 Marne-la-Vallée, France \\ ${ }^{2}$ Univ Gustave Eiffel, Univ Paris Est Creteil, CNRS, MSME UMR 8208, F-77454 Marne-la-Vallée, France
}

\begin{abstract}
:
This article presents an experimental and numerical parametric study of the acoustical properties of monodisperse open-cell solid foam. Solid foam samples are produced with very good control of both the pore size (from 0.2 to $1.0 \mathrm{~mm}$ ) and the solid volume fraction (from $6 \%$ to $35 \%$ ). Acoustical measurements are performed by the threemicrophone impedance tube method. From these measurements, the visco-thermal parameters-namely, viscous permeability, tortuosity, viscous characteristic length, thermal permeability, and thermal characteristic length—are determined for an extensive number of foam samples. By combining Surface Evolver and finite-element method calculations, the visco-thermal parameters of body centered cubic (bcc) foam numerical samples are also calculated on the whole range of solid volume fraction (from $0.5 \%$ to $32 \%$ ), compared to measured values and to theoretical model predictions [Langlois et al. (2019). Phys. Rev. E 100(1), 013115]. Numerical results are then used to find approximate formulas of visco-thermal parameters. A systematic comparison between measurements and predictions of the Johnson-Champoux-Allard-Lafarge (JCAL) model using measured visco-thermal parameters as input parameters, reveals a consistent agreement between them. From this first step, a calculation of the optimal microstructures maximizing the sound absorption coefficient is performed. (C) 2020 Acoustical Society of America.
\end{abstract} https://doi.org/10.1121/10.0001995

(Received 27 April 2020; revised 17 August 2020; accepted 3 September 2020; published online 29 September 2020)

[Editor: Kirill V. Horoshenkov]

Pages: $1767-1778$

\section{INTRODUCTION}

Foam is a dispersion of gas in a liquid or solid matrix. Its structure consists of membranes, ligaments (intersection of three membranes), and vertices (intersection of four ligaments) (Fig. 1). Whereas closed membranes are necessary to ensure the mechanical stability of liquid foam (Cantat $e t$ al., 2013), they can be open in solid foam, allowing for the foam cells (pores) to be connected through windows.

Solid foams find applications in many fields, such as mechanical dampers, thermal, or/and acoustic insulation heat exchangers. Therefore, numerous works are still devoted to elucidate the link between their microstructure and their macroscopic properties, such as thermal, acoustical, mechanical, and transport properties (Despois and Mortensen, 2005; Doutres et al., 2011; Gibson and Ashby, 1997; Jang et al., 2008; Kumar and Topin, 2014). Recently, mineral solid foams, made from cement or geopolymer or gypsum, have been studied for their applications as building materials, requiring a good compromise between mechanical strength, and sound and/or thermal insulation (Chevillotte et al., 2013; Feneuil et al., 2019; Hung et al., 2014; Kaddami and Pitois, 2019; Zhang et al., 2014). This kind of solid foam differs from polymeric foams, which are

\footnotetext{
a)Electronic mail: vincent.langlois@univ-eiffel.fr, ORCID: 0000-00033633-4144.

b) ORCID: 0000-0001-7796-2118.
}

widely used for sound and/or thermal insulation by both their wide range of porosity (from 0.65 to 0.95 ) and their highly stiff porous frames.

In acoustics of porous media, semi-phenomenological models, such as the Johnson-Champoux-Allard-Lafarge (JCAL) model (Champoux and Allard, 1991; Johnson et al., 1987; Lafarge et al., 1997), link the frequency-dependent macroscopic acoustic behavior to visco-thermal parameters resulting from the microstructure. These visco-thermal parameters were introduced to properly describe the asymptotic behaviors at high and low frequencies of the dynamic effective density for viscous parameters, and of the dynamic bulk modulus for thermal parameters. For the JCAL model, the viscous parameters are the static viscous permeability $k_{0}$, the high frequency tortuosity $\alpha_{\infty}$ and the viscous characteristic length $\Lambda$, and the thermal parameters are the thermal permeability $k_{0}^{\prime}$ and the characteristic length $\Lambda^{\prime}$. Numerous studies have modeled these visco-thermal parameters by using upscaling methods for various microstructural configurations. The visco-thermal parameters of idealized open-cell foams, made of 14-sided cells having spheres for vertices and circular cylinders for ligaments, were calculated by finite-element method (FEM) with the aim of predicting the acoustic behavior of highly porous foam (Perrot et al., 2012). The effects on foam acoustical properties of the membrane aperture size separating neighboring pores were studied by using models for visco-thermal parameters (Doutres et al., 2011, 2013), or FEM calculations (Hoang 


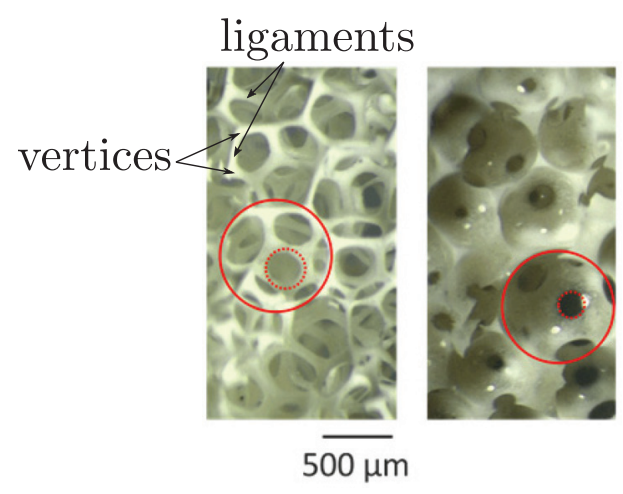

FIG. 1. (Color online) Close-up of foam samples revealing the open-cell microstructure and showing pores (continuous lines) and windows apertures (dotted lines). Left: Pore diameter $D_{p} \approx 800 \mu m, \phi_{s} \approx 0.08$; right: Pore diameter $D_{p} \approx 800 \mu m, \phi_{s} \approx 0.3$.

and Perrot, 2012; Trinh et al., 2019). However, although the visco-thermal parameters can be characterized experimentally (Olny and Panneton, 2008; Panneton and Olny, 2006), many numerical studies have no systematic experimental data that can be compared with their predictions. Moreover, such theoretical studies are based on very idealized pore microstructure, which is questionable when it comes to assess the behavior of real materials. Thus, the use of more realistic microstructures should be preferred for the calculation of visco-thermal parameters, and in particular in the context of researching the optimal microstructure maximizing the sound absorption (Chevillotte and Perrot, 2017). To achieve this task, the software "Surface Evolver" (SE) turns out to be an excellent tool, because it has proven possible to reproduce the foam microstructure produced from gas dispersion within a fluid (Jang et al., 2008; Kraynik et al., 2003, 2004).

The present paper deals with the acoustical properties of monodisperse open-cell foams (i.e., all pores have the same size, and foam contains no membrane). Numerical simulations combining SE and FEM are used to calculate the visco-thermal parameters of body centered cubic (bcc) foam samples (Fig. 2). In contrast to previous studies, these calculations are systemically compared to measurements performed on real foam samples.

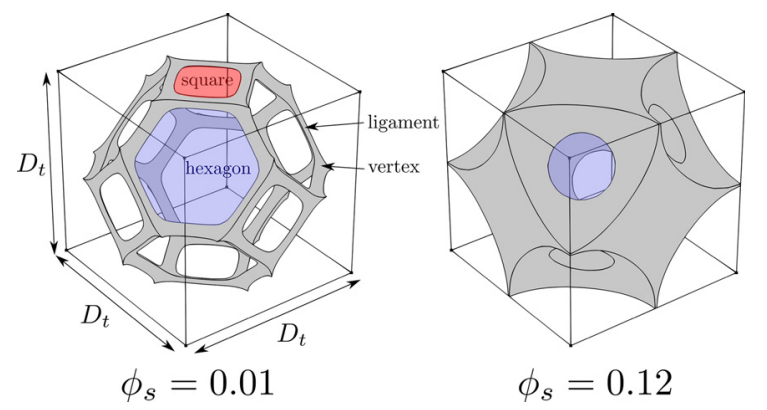

FIG. 2. (Color online) Bcc foam PUC as predicted by Surface Evolver for two solid volume fractions. Membranes separating the two neighboring bubbles were removed. Note that at low solid volume fraction $\left(\phi_{s} \lesssim 0.11\right)$, the PUC has two kinds of windows: square- and hexagon-like windows. And when $\phi_{s} \gtrsim 0.11$, only the original hexagon-like windows remain open.
This paper is organized as follows. In Sec. II, methods used to produce real foam and to generate numerical samples are presented, as well as the comparison of their respective microstructures. Section III is devoted to the visco-thermal parameters, where a comparison between experimental and numerical values is presented. The results are discussed and compared to dedicated theoretical models. In Sec. IV, we focus on the acoustic properties and sound absorption coefficient $(S A C)$ especially. The JCAL model predictions of SAC are compared to experimental measurements. Finally, calculations of the optimal microstructures maximizing the sound absorption coefficient average predicted by the JCAL model are presented.

\section{REAL SOLID FOAM AND NUMERICAL SAMPLES: PRODUCTION AND MICROSTRUCTURE CHARACTERIZATION}

\section{A. Monodisperse geopolymer foam}

A millifluidic setup, described in detail in Kaddami and Pitois (2019), is used to generate monodisperse foam (Fig. 3). Based on two main steps, it is able to control both the bubble size and the gas volume fraction separately. In the first step, a precursor aqueous dry foam is formed by forcing a surfactant solution flow and a nitrogen gas flow to converge in a small T-junction. The size of bubbles is fixed by adjusting the relative flow rates of solution and gas. The precursor foam is stored in a vertical glass column where a controlled foam drainage occurs. At the column exit, the gas volume fraction $\phi_{g}$ is around $99 \%$. In a second step, a metakaolin suspension is mixed with the precursor foam in a second $\mathrm{T}$-junction. The final gas volume fraction is fixed by adjusting the relative flow rates of the metakaolin suspension and precursor foam. At the exit of the second Tjunction, the metakaolin fresh foam is introduced in a mold. When filled, the mold is closed to prevent water evaporation and, the foam samples are placed at $20^{\circ} \mathrm{C}$ for a week. The

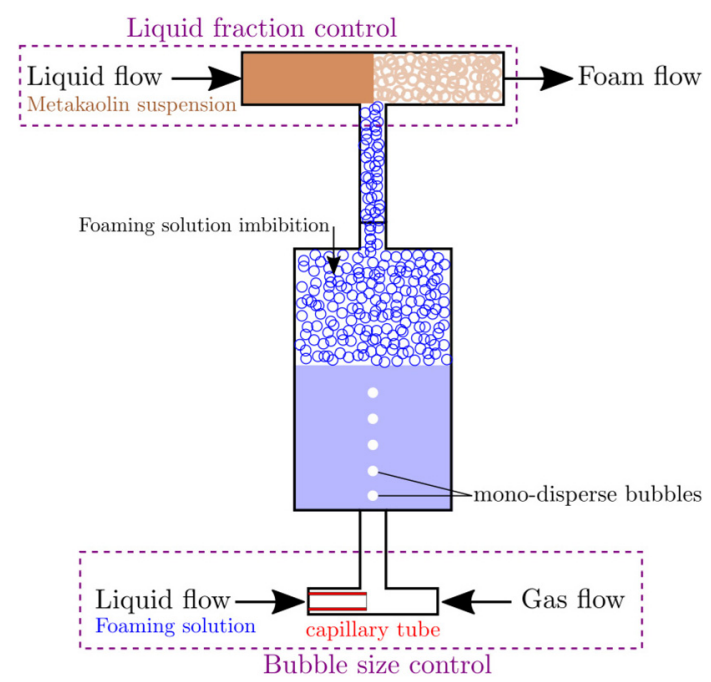

FIG. 3. (Color online) Scheme of the foaming process used to produce the geopolymer foam samples. 
solid foam sample can then easily be removed from its mold. In that stage, the films separating neighboring bubbles in foam are breaking as evidenced by a characteristic emission noise and by a binocular microscope observation (Fig. 1). To remove an amount of the initial water still present in the foam pore space after the metakaolin geopolymer setting, foam samples must be dried. After drying, acoustical measurements are performed, and the microstructure of the geopolymer foam samples is characterized by binocular microscope observations and for a few samples by x-ray microtomography.

Ensuring foam stability is a key point during each step of the whole process. Concerning the precursor foam production, its duration depends on the size of the bubbles, the smaller the bubbles are, the longer the time of production is. To prevent the ripening in the case of the smallest sizes $(0.2$ and $0.3 \mathrm{~mm}$ ), nitrogen used as bubbling gas in the precursor foam production was saturated in perfluorohexane vapor (Gandolfo and Rosano, 1997). To ensure the stability of the fresh foam before the geopolymer setting, particular attention was paid to the choice of geopolymer formulation, as shown in detail in Kaddami and Pitois (2019). However, despite our special care, a small and unexpected drainage was observed for high solid volume fraction $\left(\phi_{s} \gtrsim 0.3\right)$ leading to a low gradient of solid volume fraction.

Open-cell foams with monodisperse pore diameter $D_{p}$ ranging from 0.2 to $1.0 \mathrm{~mm}(0.2,0.3,0.6,0.8$, and $1.0 \mathrm{~mm})$ and solid volume fraction ranging from 0.1 to 0.38 were thus produced. X-ray microtomography image analysis shows that the relative standard deviations for the pore diameter are around $5 \%$ for the smallest diameters and up to $15 \%$ for the largest ones.

\section{B. PUC numerical samples}

As shown in Fig. 2, a periodic unit cell (PUC) is used to represent the pore structure in foam samples. The cell is based on the bcc paving. The unit cube of size $D_{t}$ includes two pores (Fig. 2): one located in the center of the cube, and the other split into eight parts at the vertices of the cube. The SEF-FIT software, which runs SE as computational engine (Brakke, 1992), was used to compute the shapes of bcc foam samples by minimizing their surface tension energy. The fine and deep convergence algorithms within SEF-FIT were used to approach the shape of the equilibrium foam surfaces. Before the last and deepest convergence procedure, an edge length threshold equal to $0.02 D_{t}$ was used (i.e., all the edge lengths are smaller than this length threshold) leading to a mean edge length of about $(0.012 \pm 0.002) D_{t}$. After the SE calculations, membranes separating the neighboring pores were removed to obtain a fully open-cell foam. Figure 2 shows two examples of solid pore shapes obtained at $\phi_{s}=0.04$ and 0.12 . For low solid volume fraction $\left(\phi_{s} \lesssim 0.11\right)$, the structure corresponds to the so-called Kelvin structure (i.e., each bubble is linked to 14 bubbles through two kinds of windows: square-like windows and hexagon-like windows). The aperture size is defined by an equivalent diameter of window aperture, $d_{o}=\left((4 / \pi) S_{o}\right)^{1 / 2}$, where $S_{o}$ is the surface area of the membrane predicted by SEF-FIT calculations. The aperture sizes of both types of window, $d_{o, s q}$ for square-like windows and $d_{o, h e x}$ for hexagon-like windows, are given in Fig. 4. As the solid volume fraction increases, the aperture sizes of the windows decrease, leading to a closure of square-like windows near to $\phi_{s} \approx 0.11$. The full closure of the original hexagon-like windows, leading to closed-cell foam (when $\phi=\phi_{s}^{\star}$, the critical solid volume fraction), is reached when the pore becomes spherical for $\phi_{s}=\phi_{s, B C C}^{\star}=1-(\sqrt{3} / 8) \pi$ $\approx 0.32$ (for bcc packing of spheres). These values $\left(\phi_{s} \approx 0.11\right.$ and $\phi_{s} \approx 0.32$ ) are in agreement with previous results found by Murtagh et al. (2015).

We define a bubble size (or pore size) $D_{p}$ as the diameter of a sphere having the same volume as the bubble: $D_{p}$ $=\left((3 / \pi)\left(1-\phi_{s}\right)\right)^{1 / 3} D_{t}$.

\section{Microstructure of real foam samples compared to numerical bcc foam}

In addition to solid volume fraction and pore size, the mean size $\left\langle d_{o}\right\rangle$ of apertures between pores and the mean number $N_{v}$ of open windows per pore are important microstructural parameters for macroscopic properties such as permeability and tortuosity (Despois and Mortensen, 2005; Johnson et al., 1987; Kirkpatrick, 1973; Langlois et al., 2018 , 2019). For some real foam samples, binocular microscope observations were used to determine the distribution of the aperture sizes. For each sample, around fifty apertures were observed in order to calculate the mean aperture $\left\langle d_{o}\right\rangle_{S_{o}}$. Figure 4 shows the evolution of the weighted mean aperture as a function of the solid volume fraction. As shown for the numerical geometries, the aperture size decreases as the solid volume fraction increases. However, for real samples, open windows were observed for the solid volume fraction higher than $\phi_{s, B C C}^{\star}$. Due to the small

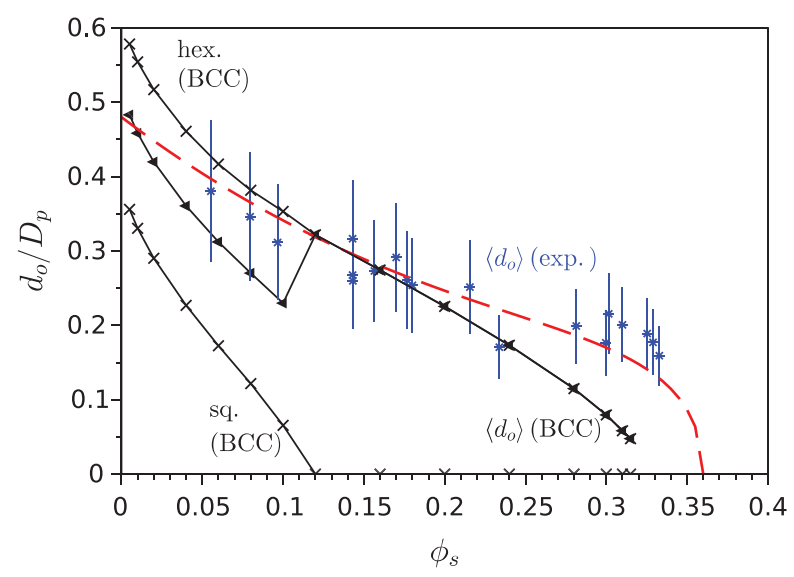

FIG. 4. (Color online) Diameter of apertures $d_{o}$ divided by the pore diameter $D_{p}$ as a function of the solid volume fraction $\phi_{s}$. The dashed line is drawn by using Eq. (1). The error bars correspond to the standard deviation. Note that for bcc numerical samples, the aperture size of hexagon- and square-like windows and the mean aperture size $\left\langle d_{o}\right\rangle$ are distinguished. The aperture diameter is defined as the diameter of a disk having the same area as the membrane area $S_{o}$ closing the windows after SE calculations: $d_{o, i}=\left((4 / \pi) S_{o, i}\right)^{1 / 2}$, where index $i$ is associated to hexagon- or square-like windows. 
drainage detected in real foam samples for high solid volume fraction, an accurate measurement of the critical solid volume fraction $\phi_{s}^{\star}$ is not possible. However, the results of our experiments suggest that $\phi_{s, \exp }^{\star} \approx 0.38$. This value is close to the common value found in the literature $\phi_{s}^{\star} \approx 0.36$ for monodisperse and disordered foam (Drenckhan and Hutzler, 2015). Based on our data presented in Fig. 4, we propose the following expression to describe the measured aperture size over the full range of solid volume fraction:

$$
\begin{aligned}
\left\langle d_{o}\right\rangle_{S_{o}}= & D_{p}\left\{\left(\phi_{s}^{\star}-\phi_{s}\right)^{0.5}\left(1-\left(\phi_{s}^{\star}-\phi_{s}\right)\right)^{0.5}\right. \\
& \left.-3.75 \phi_{s}\left(\phi_{s}^{\star}-\phi_{s}\right)\right\} .
\end{aligned}
$$

For few samples, an analysis of x-ray microtomography images using ImageJ software, described in detail in Pitois et al. (2020), allowed us to identify the pores (their centers and their sizes) and to locate the apertures between them. To check the location of the apertures, a calculation is performed by testing for each pair of adjacent pores if an aperture was detected in the vicinity of its expected position (defined by the barycenter of the pore centers weighted by the pore sizes). If such an aperture is found in the list of "aperture" objects, this aperture is considered as being identified. At the end of the identification process, the mean number $N_{v}$ of open windows per pore is calculated. Figure 5 shows the mean number of open windows per pore found for four samples. Experimental values are close to the values determined from numerical simulations, showing a decrease of $N_{v}$ as the solid volume fraction increases. Our experimental data for $N_{v}$ are few but they can be combined with expected bounds [i.e., $N_{v} \approx 14$ for $\phi_{s} \approx 0$ (Kelvin cells) and $N_{v} \approx 6$ for $\left.\phi_{s} \approx \phi_{s, \text { exp }}^{\star}\right]$. Figure 5 shows that $N_{v}\left(\phi_{s}\right)$ can be described by the following linear relation:

$$
N_{v}=8\left(1-\frac{\phi_{s}}{\phi_{s}^{\star}}\right)+6 .
$$

Finally, the analysis of the pore positions by computing the pair distribution function did not reveal any order in our

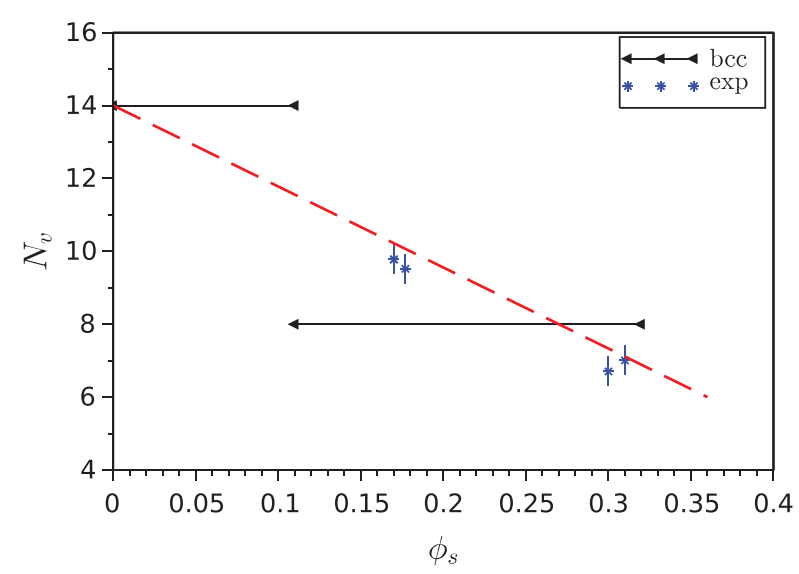

FIG. 5. (Color online) Mean number of apertures per pore $N_{v}$ as a function of the solid volume fraction $\phi_{s}$. The dashed line is drawn by using Eq. (2). Note that for bcc foam, the discontinuity of $N_{v}$ at $\phi_{s} \approx 0.11$ is due to the closure of the six original square-like windows. samples, contrary to the bcc numerical samples. Therefore, except for this major difference between real foam samples and bcc numerical samples, the microstructure of SE bcc numerical samples appears quite similar to that of the real foam samples in terms of $N_{v}$ and $d_{o}$ behavior (except for $\phi_{s} \approx 0.32$ ).

\section{VISCO-THERMAL PARAMETERS}

\section{A. FEM calculations on PUC samples}

In this section, we briefly introduce the boundary value problems used for computing the visco-thermal parameters: (1) viscous parameters: the static viscous permeability $k_{0}$, the high frequency tortuosity $\alpha_{\infty}$, and the viscous characteristic length $\Lambda$, (2) thermal parameters: the static thermal permeability $k_{0}^{\prime}$ and the thermal characteristic length $\Lambda^{\prime}$. The solution of the boundary value problem is obtained through the FEM using COMSOL Multiphysics software.

In what follows, the distinction between the viscous flow and the inertial flow is whether $\omega \ll \omega_{c}$ or $\omega \gg \omega_{c}$, respectively, with $\omega_{c}=\mu \phi_{g} /\left(\rho_{0} \alpha_{\infty} k_{0}\right)$, where $\mu$ is the dynamic viscosity of the fluid, $\rho_{0}$ is the fluid density at rest, and $\phi_{g}$ is the gas volume fraction (Pride et al., 1993).

\section{Viscous flow}

The low Reynolds number flow of an incompressible Newtonian fluid is governed by the usual Stokes equations in the fluid phase:

$$
\begin{aligned}
& \mu \Delta \boldsymbol{v}-\nabla p=0 \text { with } \nabla \cdot \boldsymbol{v}=0 \text { in } \Omega_{f}, \\
& \boldsymbol{v}=0 \text { on } \partial \Omega_{p}, \\
& \boldsymbol{v} \cdot \boldsymbol{n}=0 \text { on } \partial \Omega_{f, \text { lateral }}, \\
& p_{\text {bottom }}-p_{\text {top }}=\Delta P \text { on } \partial \Omega_{f, \text { extremety }}, \\
& \boldsymbol{v} \text { is } D_{t}-\text { periodic on } \Omega_{f},
\end{aligned}
$$

where the symbols $v$ and $p$ stand for the microscopic velocity and pressure of the fluid, respectively; $p_{\text {bottom }}$ and $p_{\text {top }}$ are the pressures on the top and bottom faces of the PUC; $\Delta P$ is the macroscopic pressure difference acting as a forcing term; and the fluid boundaries $\partial \Omega_{p}$ (pore surface), $\partial \Omega_{f, \text { lateral }}$, and $\partial \Omega_{f, \text { extremety }}$ are defined in Fig. 6.

The macroscopic pressure gradient $\lambda_{m}$ is defined as $\lambda_{m}=\Delta P / D_{t}$.

The static viscous permeability $k_{0}$ is calculated from the microscopic velocity averaged over the pore fluid volume, $\langle\mathbf{v}\rangle_{\Omega_{f}}$,

$$
k_{0}=\mu \frac{\langle\mathbf{v}\rangle_{\Omega_{f}} \cdot \mathbf{e}}{\lambda_{m}},
$$

where $\mathbf{e}$ is the unit vector pointing in the direction of the macroscopic pressure gradient, and $\langle\cdot\rangle_{\Omega_{f}}$ denotes averaging over the pore fluid volume $\langle\cdot\rangle_{\Omega_{f}}=\left(1 / V_{f}\right) \int_{\Omega_{f}} d V_{f}$. 


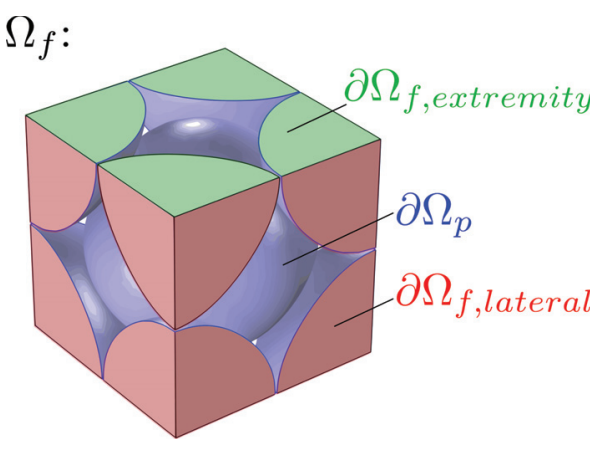

FIG. 6. (Color online) Geometry of the pore volume and definitions of the fluid boundaries $\left(\phi_{s}=0.12\right)$.

\section{Inertial flow}

At the high frequency range, the viscous boundary layer becomes negligible, and the fluid tends to behave as a perfect fluid (or inviscid fluid) and to flow through the pore space as electric charges would migrate through the pore space if it was filled by a conducting fluid having a constant conductivity. Consequently, the perfect incompressible fluid formally behaves according to the electrical conduction problem (Brown, 1980; Johnson et al., 1987):

$$
\begin{aligned}
& \Delta \varphi=0 \text { with } \mathbf{E}=-\nabla \varphi \text { in } \Omega_{f}, \\
& \mathbf{E} \cdot \mathbf{n}=0 \text { on } \partial \Omega_{p} \text { and } \partial \Omega_{f, \text { lateral }}, \\
& \varphi_{\text {bottom }}=-\varphi_{\text {top }}=-\Delta V / 2 \text { on } \partial \Omega_{f, \text { extremity }}, \\
& \varphi \text { is } D_{t}-\text { antiperiodic on } \Omega_{f},
\end{aligned}
$$

where $\mathbf{E}$ and $\varphi$ are the local electric field and the electric potential, respectively, and $\mathbf{n}$ is the unit vector normal to $\partial \Omega_{p}$ and $\partial \Omega_{f, \text { lateral }}$.

The high frequency tortuosity $\alpha_{\infty}$ and the viscous characteristic length $\Lambda$ are calculated as follows (Cortis et al., 2003; Johnson et al., 1987):

$$
\begin{aligned}
& \alpha_{\infty}=\frac{\langle\mathbf{E} \cdot \mathbf{E}\rangle_{\Omega_{f}}}{\langle\mathbf{E}\rangle_{\Omega_{f}} \cdot\langle\mathbf{E}\rangle_{\Omega_{f}}}=\frac{1}{\langle\mathbf{E}\rangle_{\Omega_{f}} \cdot \mathbf{e}}, \\
& \Lambda=\frac{2 \int_{\Omega_{f}} \mathbf{E} \cdot \mathbf{E} d V_{f}}{\int_{\partial \Omega_{p}} \mathbf{E} \cdot \mathbf{E} d S_{p}},
\end{aligned}
$$

where e corresponds to an unit global (i.e., external, locally constant) electric field.

Because of the sharp edge existing at the level of the throat between two neighboring pores [Figs. 7(a) and 7(b)], the electric field has a singularity along the edge. To reduce the lack of accuracy in the numerical approximation of $\mathbf{E}$, special care was taken to refine the mesh in the region of the sharp edge (Cortis et al., 2003; Firdaouss et al., 1998). The maximum element size $h$ along the throat edge was imposed [Fig. 7(c)]. Therefore, this size corresponds to the spatial resolution of our FEM calculations (i.e., our FEM results are similar to those with a rounded edge of radius equal to $h$ ). (a)

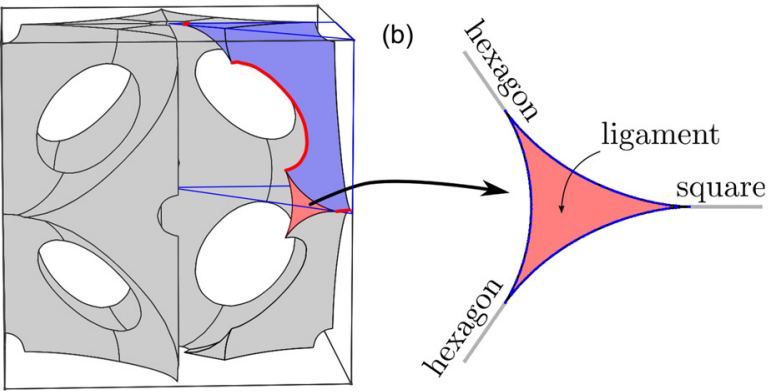

(c)

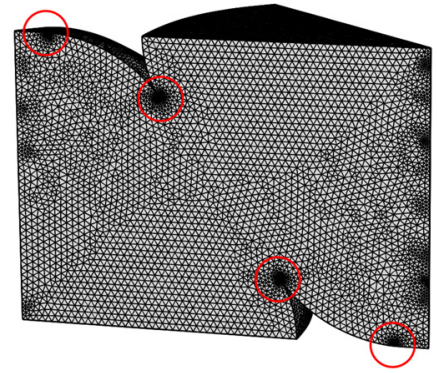

FIG. 7. (Color online) (a) Geometry of the solid frame. The edges of throats are highlighted by a red thick line. (b) Cross-section of a ligament. (c) Pore volume mesh of 1/16 Kelvin cell for FEM calculation of the inertial flow $\left(\phi_{s}=0.08\right)$.

As we are interested in geopolymer foam made from micrometric metakaolin particles and having millimetric pores, we choose $h=D_{t} / 1000$.

\section{Thermal parameters}

The static thermal permeability is given by Henry and Allard (1997) and Lafarge et al. (1997):

$$
k_{0}^{\prime}=\left(1-\phi_{s}\right)\langle\theta\rangle_{\Omega_{f}},
$$

where $\theta$ corresponds to the scaled excess temperature field and solves the following equations:

$$
\begin{aligned}
& \triangle \theta=-1 \text { in } \Omega_{f}, \\
& \theta=0 \text { on } \partial \Omega_{p}, \\
& \nabla \theta \cdot \mathbf{n}=0 \text { on } \partial \Omega_{f, \text { lateral }} \text { and } \partial \Omega_{f, \text { extremity }} .
\end{aligned}
$$

The thermal characteristic length is a geometric parameter, depending on the shape of the pore space:

$$
\Lambda^{\prime}=\frac{2 \int_{\Omega_{f}} d V_{f}}{\int_{\partial \Omega_{p}} d S_{p}} .
$$

It is to be noted that when the solid volume fraction is high enough, the pores are almost spherical. In such a case, the thermal characteristic length and the static thermal permeability can be calculated by the following formulae, $\lim _{\phi_{s} \rightarrow \phi_{s}^{\star}} \Lambda^{\prime} / D_{p}=\frac{1}{3} \quad$ and $\quad \lim _{\phi_{s} \rightarrow \phi_{s}^{\star}} k_{0}^{\prime} /\left(1-\phi_{s}\right) D_{p}^{2}=\frac{1}{60}$ (details are given in Appendix C). 


\section{B. Acoustical characterization of real foam samples}

We determined the macroscopic parameters of real foam samples by acoustic measurements performed in a three-microphone impedance tube (length: $1 \mathrm{~m}$, diameter: $40 \mathrm{~mm}$ ) (Doutres et al., 2010). The tested frequency range lies between 4 and $4500 \mathrm{~Hz}$, with a step size of $4 \mathrm{~Hz}$. The three-microphone impedance tube method consists in measuring the pressure transfer functions between the microphones and leads, after calculations, to the dynamic density $\rho_{e q}$ and the dynamic bulk modulus $K_{e q}$ of the equivalent fluid medium. The sound absorbing coefficient at normal incidence $S A C N I$ directly derives from $\rho_{e q}$ and $K_{e q}$. Based on the measured frequency dependent response functions $\rho_{e q}$ and $K_{e q}$, Panneton and Olny (2006; Olny and Panneton, 2008) proposed an inverse characterization method to estimate the visco-thermal parameters of porous materials. This characterization method requires the porosity value and the static viscous permeability $k_{0}$ as input parameters. However, by using the approximate but robust JCAL semiphenomenological model (Champoux and Allard, 1991; Johnson et al., 1987; Lafarge et al., 1997), it is possible to determine the static viscous permeability $k_{0}$. Indeed, the JCAL model predicts that the imaginary part of $\rho_{e q}$ is given by

$$
\operatorname{Im}\left(\rho_{e q}\right)=-\frac{\mu}{k_{0} \omega}\left[\frac{1}{2}+\frac{1}{2}\left(1+\left(\frac{M}{2 \omega_{\mu}} \omega\right)^{2}\right)^{0.5}\right]^{0.5} .
$$

In the aim of finding the static viscous permeability $k_{0}$, $\omega^{\prime \prime}\left(=M / 2 \omega_{\mu}\right)$ and $k_{0}$ are chosen to fit the model to data. Details of the JCAL model are given in Appendix A.

\section{Pore-network models for permeability and tortuosity}

Recent studies proposed pore-network models for permeability and electrical conductivity of solid foam (Langlois et al., 2018, 2019). For permeability, the model considers that the fluid flow through solid foam is governed by the pressure drops that occur when the fluid flows through the windows. A local fluid flow conductance is then used to describe these pressure drops (Sampson, 1891). The model describes the foam pore-space as a network of fluid flow conductances. By solving a problem similar to the calculation of the equivalent electrical resistance of an electrical resistance network, it is possible to solve the porenetwork of fluid flow conductance and to deduce the foam permeability. This pore-network model has been successfully validated for highly porous foam having thin open membranes by comparing its predictions to PUC FEM calculations (Langlois et al., 2018). For tortuosity, the problem is more difficult to solve because the electrical conductance of solid foam does not only rely on a local mechanism, such as the local pressure drops for viscous fluid flow (Langlois et al., 2018). In this case, the whole pore volume also contributes to the overall electrical conduction. However, when the apertures are small compared to the pore size, the access resistances acting at the scale of the windows' apertures are predominant compared to the bulk electrical resistance of the pore. As a consequence, the problem for electrical conductivity is very similar to the ones for permeability. Hereafter, we recall the expressions for permeability and tortuosity (where the bulk electrical resistance of the pore is neglected) regarding foam having a bcc structure (Langlois et al., 2018, 2019):

$$
\begin{aligned}
& \frac{k_{0}}{D_{p}^{2}}=\frac{1}{24} \frac{D_{p}}{D_{t}}\left[n_{s q}\left(\frac{d_{o s s q}}{D_{p}}\right)^{3}+\frac{1}{2} n_{\text {hex }}\left(\frac{d_{o, \text { hex }}}{D_{p}}\right)^{3}\right], \\
& \frac{1}{\alpha_{\infty}} \approx \frac{1}{1-\phi_{s}} \frac{D_{p}}{D_{t}}\left[n_{s q} \frac{d_{o, s q}}{D_{p}}+\frac{1}{2} n_{\text {hex }} \frac{d_{o, h e x}}{D_{p}}\right],
\end{aligned}
$$

where $n_{s q}=2$ (while $\phi_{s} \lessgtr 0.11,0$ afterward) and $n_{\text {hex }}=4$ correspond to the numbers of square- and hexagon-like windows through which the flow effectively occurs in bcc foam.

Note that the dependence of the permeability on the aperture size ( $\propto d_{o}^{3}$ involved by Sampson's law) and the one of the tortuosity to the aperture size [ $\propto d_{o}$ involved by the access resistance given by Hall's law (Hall, 1975)] come from the local conductances (or resistances) acting at the scale of the windows' apertures (Langlois et al., 2019).

When the aperture size and the pore size are in the same order of magnitude, both volume and access resistances must be taken into account to calculate the tortuosity. Here, we give the expression of tortuosity adapted for the case of bcc open-cell foam (Langlois et al., 2019):

$$
\frac{1-\phi_{s}}{\alpha_{\infty}}=\frac{\sigma_{e}}{\sigma_{f}}=2 \frac{2 g_{1} g_{2}+g_{e, h}\left(g_{1}+g_{2}\right)}{g_{1}+g_{2}+2 g_{e, h}},
$$

where $g_{1}=\left(g_{i, s h}^{-1}+2 g_{e, s}^{-1}\right)^{-1}$ and $g_{2}=2\left(g_{i, s h}+g_{i, h h}\right)$ with $g_{i, s h}$ $=0.16, \quad g_{i, h h}=0.30, \quad g_{e, i}=\left(d_{o i} / D_{t}\right)\left(1+C_{1}\left(\beta_{i}\left(d_{o i} / D_{t}\right)\right)\right.$ $\left.+C_{2}\left(\beta_{i}\left(d_{o i} / D_{t}\right)\right)^{3}\right)^{-1}$ where $\beta_{i}=\sqrt{2 \pi}$ for square windows $\left(i \equiv^{\prime} s^{\prime}\right)$ and $\left(4 \pi / 3^{1.5}\right)^{0.5}$ for hexagonal windows $\left(i \equiv^{\prime} h^{\prime}\right)$, and $C_{1}=-1.265$ and $C_{2}=0.166$.

In Eq. (14), $g_{e, s}$ and $g_{e, h}$ are the inverses of access resistances associated to square and hexagonal windows, respectively, and $g_{i, h h}$ and $g_{i, s h}$ are the electrical conductances associated to the bulk pore contribution. In the limit case $d_{o i} \rightarrow 0$, Eqs. (13) and (14) are identical.

\section{Results and discussion}

The visco-thermal parameters are gathered in Figs. 8, 9, and 10, as calculated by FEM on bcc PUC and characterized for real foam samples.

Hereafter, we consider the consistency of our numerical and experimental data in the light of theoretical models.

\section{Tortuosity and viscous permeability}

Viscous permeability $k_{0}$ and tortuosity $\alpha_{\infty}$ are two parameters governing the asymptotic behavior of the apparent dynamic density of the fluid at low frequency for $k_{0}$ and at high frequency for $\alpha_{\infty}$. As mentioned in Sec. III A, both 
JASA

https://doi.org/10.1121/10.0001995
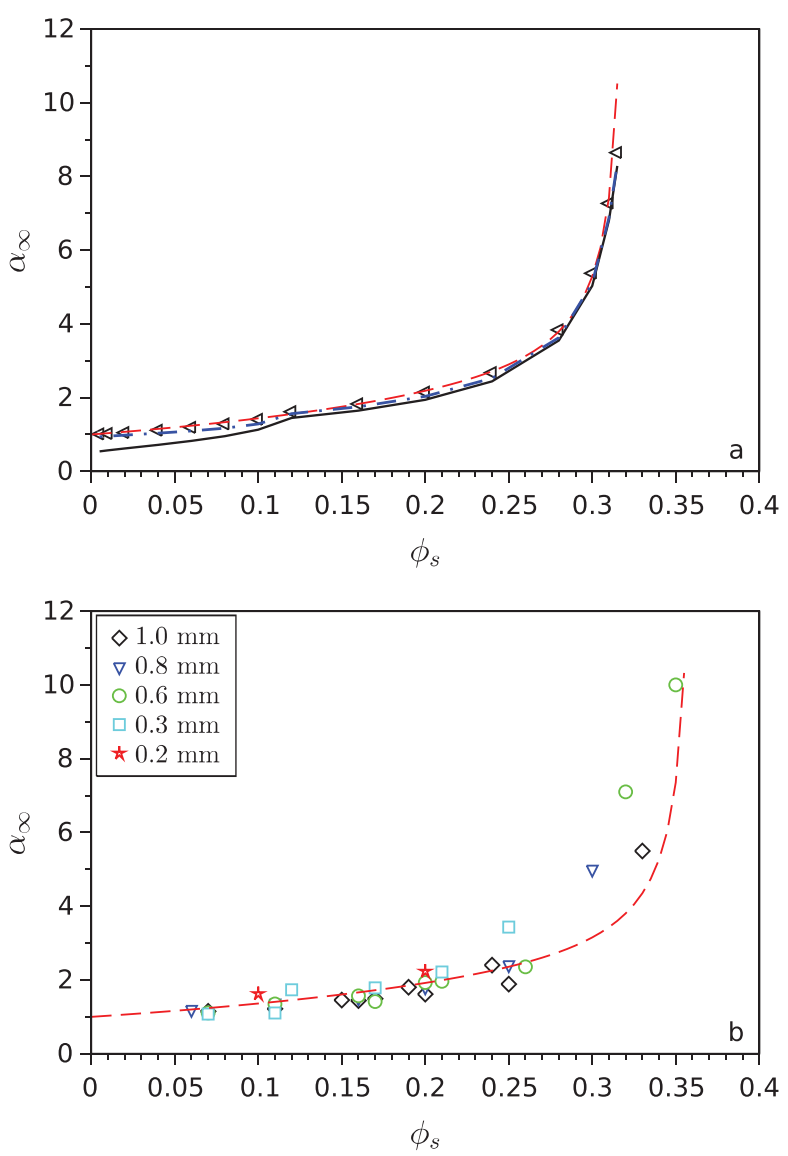

FIG. 8. (Color online) Tortuosity $\alpha_{\infty}$ as a function of the solid volume fraction $\phi_{s}$ : (a) FEM on PUC numerical samples (triangle). (b) Real foam samples for various pore sizes (markers, see legend). The red dashed lines in (a) and (b) are drawn by using Eq. (16) (with $\phi_{s}^{\star} \approx 0.32$ for bcc numerical foam samples, and $\phi_{s}^{\star} \approx 0.38$ for real foam samples). In (a), dash-dotted and full lines correspond to the pore-network model predictions with the bulk pore contribution for the blue dash-dotted line [Eq. (14)] and without for the black full line [Eq. (13)].

parameters involve a dedicated transport phenomenon: the flow of a viscous fluid at low frequency and the displacement of electric charges at high frequency, respectively. Therefore, the tortuosity at high frequency is linked to the foam electrical conductivity $\sigma_{e}$ when the pore space is filled by a fluid having an electric conductivity $\sigma_{f}: \alpha_{\infty}=$ $\left(1-\phi_{s}\right) \sigma_{f} / \sigma_{e}$ [see, for instance, Eq. 2.8 in (Johnson et al., 1987)]. Thus, as the solid volume fraction increases, the ability of fluid or electrical charges to pass through the foam samples decreases due to the progressive closure of the windows ( $\sigma_{e}$ decreases strongly when $\phi_{s}$ increases), and until vanishing when $\phi_{s} \rightarrow \phi_{s}^{\star}$. Consequently, as shown in Figs. 8 and 9, tortuosity is expected to increase when the solid volume fraction increases, while the viscous permeability decreases.

Figure 9(a) shows that the predictions of the porenetwork model for permeability is in perfect agreement with the FEM calculations except for very low solid volume fraction where a discrepancy is observed. Concerning tortuosity [Fig. 8(a)], the pore-network model predicts well the tendency calculated by FEM but leads to slightly smaller values
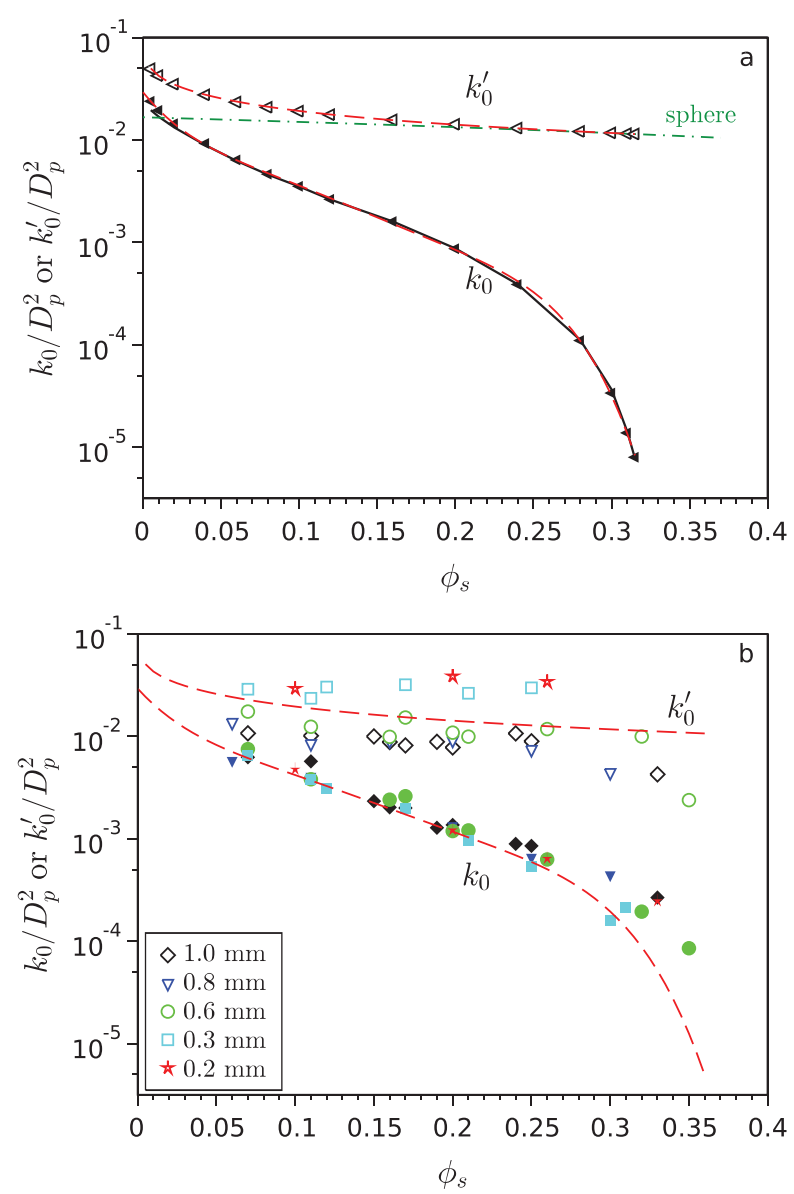

FIG. 9. (Color online) Viscous permeability $k_{0}$ and thermal permeability $k_{0}^{\prime}$ as a function of the solid volume fraction $\phi_{s}$ : (a) FEM on PUC numerical samples (triangle), (b) Real foam samples for various pore sizes (markers, see legend). The red dashed lines in (a) and (b) are drawn by using Eq. (16) (with $\phi_{s}^{\star} \approx 0.32$ for bcc numerical foam samples, and $\phi_{s}^{\star} \approx 0.38$ for real foam samples). In (a), the black full line corresponds to the pore-network model predictions: Eq. (12). Note that for spherical pores, $k_{0}^{\prime} / D_{p}^{2}$ $=\left(1-\phi_{s}\right) / 60$ (green dashed-dotted line $)$.

even at high solid volume fraction. This gap is not surprising at low solid volume fraction, because the resistance term taking into account the bulk electrical resistance should be added. Taking into account this contribution [Eq. (14)] allows us to improve the agreement [see Fig. 8(a)] while much more complexity is introduced in the model. However, a small discrepancy between FEM calculations and theoretical predictions remains at high solid volume fraction. It could be due to the effect of the sharp edge of throats, which alters the local electrical field singularity, and consequently, access resistances.

\section{Other parameters}

To our knowledge, no theoretical models have been proposed yet for the viscous characteristic length and thermal parameters of solid foam. The definitions of thermal and viscous characteristic lengths involve a ratio of two integrals over the pore volume at the numerator and over the pore surface at the denominator. In solid foam samples, the pore 

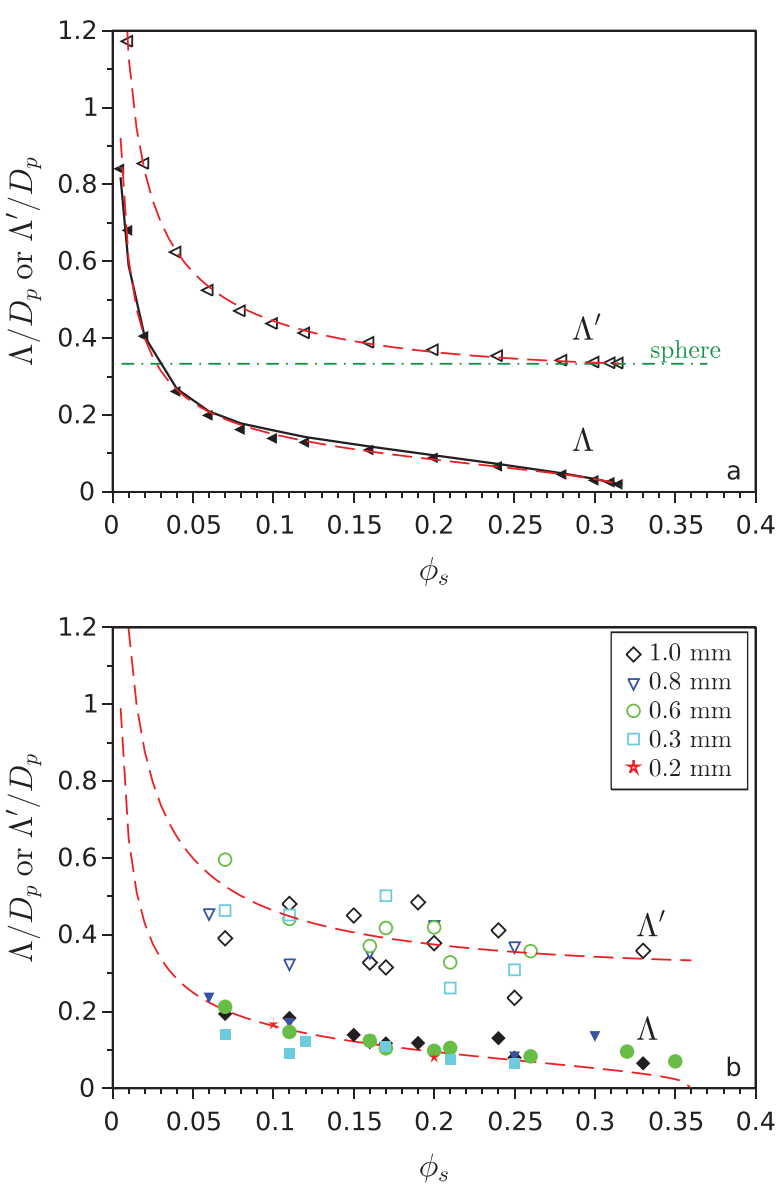

FIG. 10. (Color online) Viscous characteristic length $\Lambda$ and thermal characteristic length $\Lambda^{\prime}$ as a function of the solid volume fraction $\phi_{s}$ : (a) FEM on PUC numerical samples (triangle). (b) Real foam samples for various pore sizes (markers, see legend). The dashed lines in (a) and (b) are drawn by using Eq. (16) (with $\phi_{s}^{\star} \approx 0.32$ for bcc numerical foam samples, and $\phi_{s}^{\star}$ $\approx 0.38$ for real foam samples). In (a), full line for $\Lambda$ is drawn by using Eq. (15). Note that for spherical pores, $\Lambda^{\prime} / D_{p}=\frac{1}{3}$ (dashed-dotted line).

volume does not evolve significantly as the solid volume fraction increases compared to the pore surface: on the one hand, $\int_{\partial \Omega_{f}} d S_{f} \rightarrow 0$, when $\phi_{s} \rightarrow 0$; whereas on the other hand, $\int_{\Omega_{f}}^{\Omega_{f}} d V_{f} \rightarrow C s t$, when $\phi_{s} \rightarrow 0$ (see Figs. 1 and 2). Therefore, as shown in Fig. 10(a), each characteristic length diverges when $\phi_{s}$ tends to zero and decreases as $\phi_{s}$ increases. This result is in qualitative accordance with the expected behavior for fibrous materials. For such materials, Allard and Atalla (2009) shows that $\Lambda=\Lambda^{\prime} / 2=1 / 2 \pi R_{0} \mathcal{L}$ where $\mathcal{L}$ is the total length of fibers per unit volume of fibrous material for cylindrical fibers of radius $R_{0}=\left(\phi_{s} / \pi \mathcal{L}\right)^{0.5}$ in the dilute limit (no interaction between fibers). Therefore, for fibrous materials with a fixed $\mathcal{L}$, both characteristic lengths diverge when $\phi_{s} \rightarrow 0$. In the limit of low $\phi_{s}$, the foam microstructure is not far from the microstructure of fibrous materials as ligaments are very thin and elongated. This microstructural analogy between fibrous material and foams when $\phi_{s} \rightarrow 0$ is consistent with the ratio $\Lambda^{\prime} / \Lambda$ being close to two for both the simulated and experimental foam samples. However, the relations $\Lambda=\Lambda^{\prime} / 2=1 / 2 \pi R_{0} \mathcal{L}$, which is correct for fibrous media (in the dilute regime), are not directly applicable for foams, even when $\phi_{s} \rightarrow 0$, because of the non-circular cross-section shape of the ligaments. Moreover, for high solid volume fraction, the ratio $\Lambda^{\prime} / \Lambda$ diverges because $\Lambda^{\prime} \rightarrow \frac{1}{3} D_{p}$ ["sphere limit," Fig. 10(a)] and $\Lambda \rightarrow 0$. Therefore, a foam specific model for each characteristic length remains to be found. As the thermal characteristic length $\Lambda^{\prime}$ is a pure geometrical parameter depending on the real shape of foam pore, no simple geometric model can be used to evaluate this parameter. Numerical calculations using SE appear to be appropriate to find a realistic relationship between $\Lambda^{\prime}$ and $\phi_{s}$. A similar approach was used by Pitois et al. (2009) to calculate the volume specific surface area $S_{V}$ as a function of the liquid volume fraction $\phi_{\text {liq }}$ in the context of the study of liquid foam drainage study (note that $\left.S_{V} \equiv 2 / \Lambda^{\prime}\right)$. Concerning the viscous characteristic length $\Lambda$, its definition involves a pore-volume-to-surface ratio weighted by the square of the electrical field [cf. Eq. (7)]. As the electrical field is very intense along the sharp edge of throats (cf. Sec. III A), this area of the pore volume is favored by the weighted average procedure. Therefore, for high solid volume fraction, the viscous characteristic length is comparable to the size of the window aperture [Fig. 10(b)]. However, this is no longer applicable when the solid volume fraction is low due to the low surface area of pores. Clearly, the viscous characteristic length seems to be a balance between the size of the window aperture and the ligament thickness (or the pore surface area). These considerations lead us to propose the following expression for $\Lambda$, which was found to give a very good agreement with numerical values [see Fig. 10(a)]:

$$
\frac{\Lambda}{D_{p}} \approx \frac{D_{t}^{2}\left\langle d_{o}\right\rangle_{S_{o}}}{S_{\text {p/pore }}},
$$

where $S_{p / \text { pore }}$ corresponds to the pore surface area of a single pore (i.e., the pore surface area contained in the PUC divided by two), and $\left\langle d_{o}\right\rangle_{S_{o}}$ is the area-weighted mean diameter of apertures.

Concerning the thermal permeability, Fig. 9 shows that $k_{0}^{\prime} / D_{p}^{2} \rightarrow \frac{1}{60}\left(1-\phi_{s}\right)$ when $\phi_{s} \rightarrow \phi_{s}^{\star}$ (as expected for spherical pores). For low solid volume fraction, the thermal permeability predicted by our FEM results can be compared to the ones obtained for fibrous materials (cf. Appendix B). This model predicts that $k_{0}^{\prime} \propto-\ln \left(\phi_{s}\right)$ when $\phi_{s} \rightarrow 0$. Therefore, the thermal permeability diverges when $\phi_{s} \rightarrow 0$. For real foam samples, results are significantly dispersed around the values calculated by FEM.

\section{Approximate formulas}

For each visco-thermal parameter, a systematic comparison between FEM calculations on bcc numerical foam samples and real monodisperse foam characterization reveals a good agreement between them except for high solid volume fraction due to the discrepancy in the critical solid volume fraction $\phi_{s}^{\star}$. Approximate formulas for visco-thermal parameters are derived from our numerical results (Figs. 8, 9, and 10): 


$$
\begin{aligned}
\frac{k_{0}}{D_{p}^{2}} \approx & \exp \left(-68.617 x^{5}+148.54 x^{4}-124.36 x^{3}\right. \\
& \left.+49.897 x^{2}-13.701 x-3.532\right), \\
\frac{k_{0}^{\prime}}{D_{p}^{2}} \approx & \frac{1}{60}\left(-0.68 \ln (x)+0.48-\phi_{s}^{\star}+0.7 x-0.18 x^{2}\right), \\
\frac{\Lambda}{D_{p}} \approx & \left(0.087 x^{1.16}(1-x)^{0.37}+0.065 x^{0.37}(1-x)^{1.16}\right) x^{-1}, \\
\frac{\Lambda^{\prime}}{D_{p}} \approx & \frac{1}{3}\left(-0.85(x)^{0.94}+1.85(x)^{0.5}\right)^{-1}, \\
\alpha_{\infty} \approx & \left(1-\phi_{s}\right)\left(0.47(1-x)^{2.47}+0.53(1-x)^{0.5}\right)^{-1}, \quad(16
\end{aligned}
$$

where $x=\phi_{s} / \phi_{s}^{\star}$.

Figures 8,9 , and 10 show that these formulas can be used to estimate the values of visco-thermal parameters of monodisperse foam samples on the whole range of $\phi_{s}$ by choosing the appropriate critical solid volume fraction $\phi_{s}^{\star}$ $\left(\phi_{s}^{\star} \approx 0.32\right.$ for bcc numerical foam samples, and $\phi_{s}^{\star} \approx 0.38$ for real foam samples). These formulas are only valid for the case of monodisperse open-cell foams, because their dispersions in pore sizes, aperture size, and number of apertures per pore are low. Note that the formula for $\Lambda$ is established for a maximum element size $h$ (spatial resolution of the throat edge) equal to $0.001 D_{t}$. For other spatial resolutions, our FEM calculations performed with various values of $h / D_{t}$ and $\phi_{s}=0.2$ show that a correction must be applied to better predict $\Lambda: \Lambda\left(x, h / D_{t}\right) / D_{p} \approx \Lambda(x, 0.001) /$ $\left(1-0.103 \log \left(h / D_{t}\right)\right)$. These formulas could be useful in the context of the characterization of visco-thermal parameters of foam samples, or in the context of material optimization, as illustrated in Sec. IV.

\section{NORMAL INCIDENCE SOUND ABSORPTION}

The present section focuses on the sound absorption of foam materials by presenting our measurements performed on real foam samples and by comparing them to the predictions of the JCAL model. The JCAL model is one of the most popular semi-phenomenological models of the acoustic properties of rigid porous materials. The constitutive equations of this model are recalled in Appendix A. The JCAL model agrees closely with the experimental data of dynamic bulk modulus $K_{e q}$ and dynamic density $\rho_{e q}$ of the equivalent fluid medium (see supplementary material). ${ }^{1}$ At the end of this section, by using the previously established approximate formulas to calculate the input parameters of the JCAL model, an optimization calculation of normal incident sound absorption coefficient $S A C_{N I}$ will be presented.

Figure 11 shows that foam samples of thickness between 20 and $30 \mathrm{~mm}$ with low solid volume fraction and a pore size around $0.3 \mathrm{~mm}$ have high sound absorption coefficients on a large frequency range. However, as sound absorption depends on both the pore size and the solid volume fraction, other configurations on $\phi_{s}$ and $D_{p}$ leading to high sound absorption could be possible (at a given thickness, with a rigid backing). Predicting the effect of each
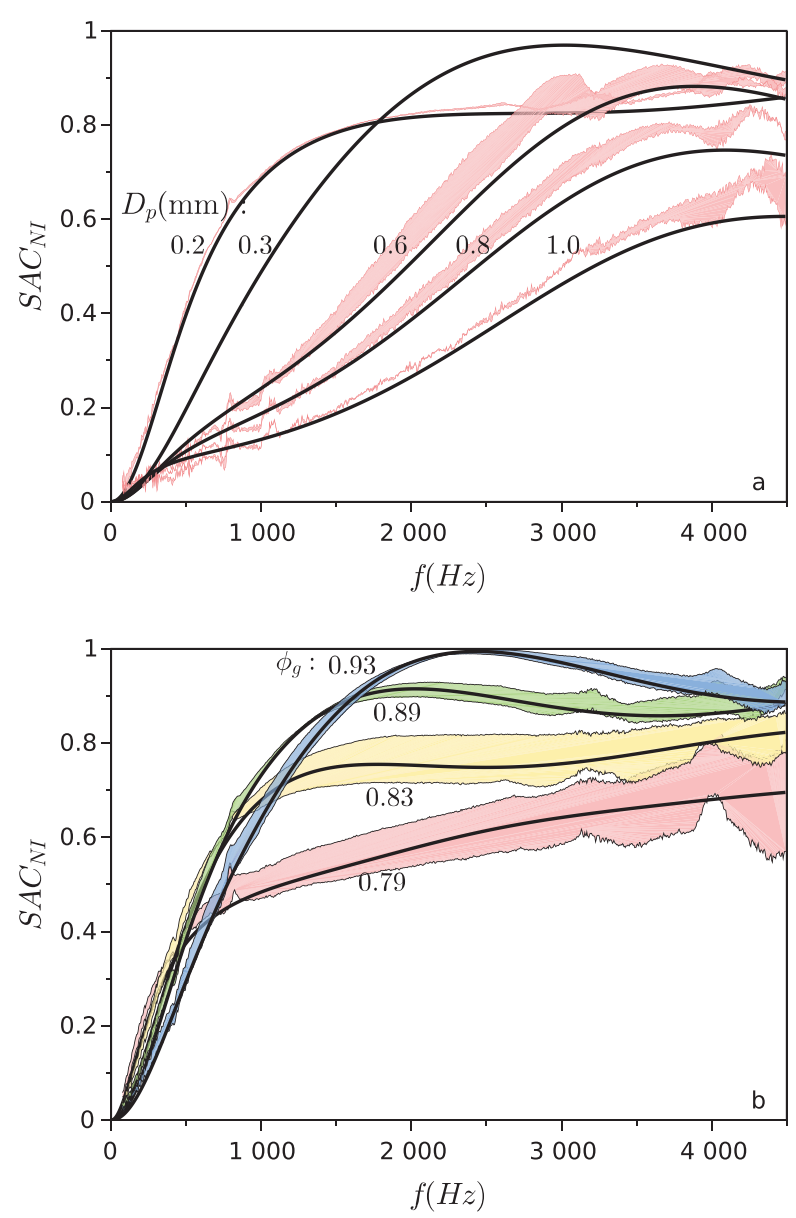

FIG. 11. (Color online) Sound absorption coefficient at normal incidence $S A C_{N I}$ as a function of frequency for real foam samples: (a) various pore sizes $D_{p}$ with $\phi_{s} \approx 0.1$ (sample thickness $19 \mathrm{~mm}$ ), (b) various solid volume fraction with $D_{p} \approx 0.3 \mathrm{~mm}$ (sample thickness $28 \mathrm{~mm}$ ). Full lines correspond to the JCAL predictions plotted by using the characterized parameters. In (a), the measurement for $D_{p}=0.3 \mathrm{~mm}$ is omitted because the sample thickness was $28 \mathrm{~mm}$.

parameter on SAC is not straightforward. For example, Fig. 11(a) shows that the maximal value of the sound absorption coefficient does not depend monotically on the pore size at constant solid volume fracion $\phi_{s}$ (i.e., at constant ratio $\left.\left\langle d_{o}\right\rangle_{S_{0}} / D_{p}\right)$. Indeed, the sound absorption coefficient measures the amount of energy, which is backward in the incident direction when the material is backed by an impervious rigid wall. Therefore, this coefficient depends on the ability of the incident sound to pass through the first air/ foam interface and on the ability of the porous material to dissipate the sound energy. A highly porous material favors the first mechanism but not the second. Thus, a good balance between these two mechanisms has to be found to maximize the sound absorption. Well-validated theoretical models are therefore expected to be helpful in finding the optimal configuration for $\phi_{s}$ and $D_{p}$. Figure 11 shows that the predictions of the JCAL model may be quite relevant to pursuing this objective. In order to provide a global indicator of the $S A C_{N I}$ over a large frequency range, we use an average of $S A C_{N I}$ by one-third octave bands $f_{i}$ from 125 to $4000 \mathrm{~Hz}$ : 


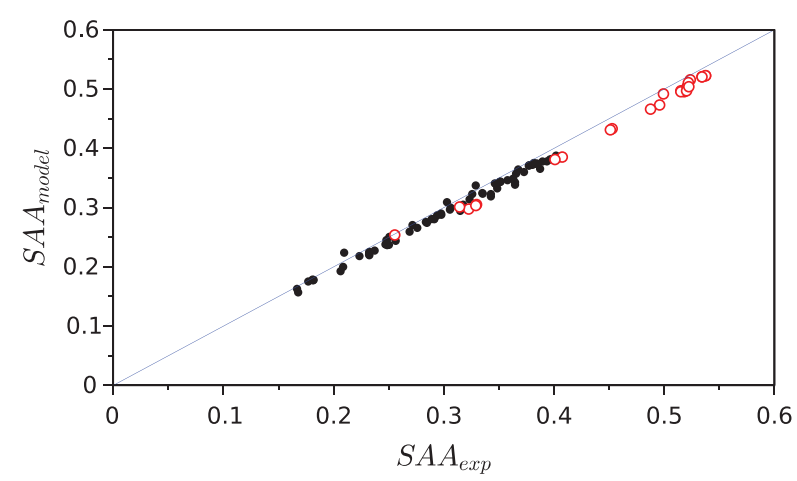

FIG. 12. (Color online) Comparison between the sound absorption averages measured on real foam samples, $S A A_{\text {exp }}$, and the ones predicted by the JCAL model, $S A A_{\text {model }}$. Filled dots correspond to $19-\mathrm{mm}$ thick samples, and empty dots to 28 -mm thick samples.

$$
S A A=\frac{1}{16} \sum_{f_{i}} S C A_{N I, 1 / 3}\left(f_{i}\right)
$$

Note that the standard ASTM C423 (ASTM C423-17, 2017) uses a smaller frequency range $(200-2500 \mathrm{~Hz})$.

Figure 12 compares the mean sound absorption coefficient $S A A$ measured on real foam samples to the ones calculated with the JCAL model. It appears that the JCAL model

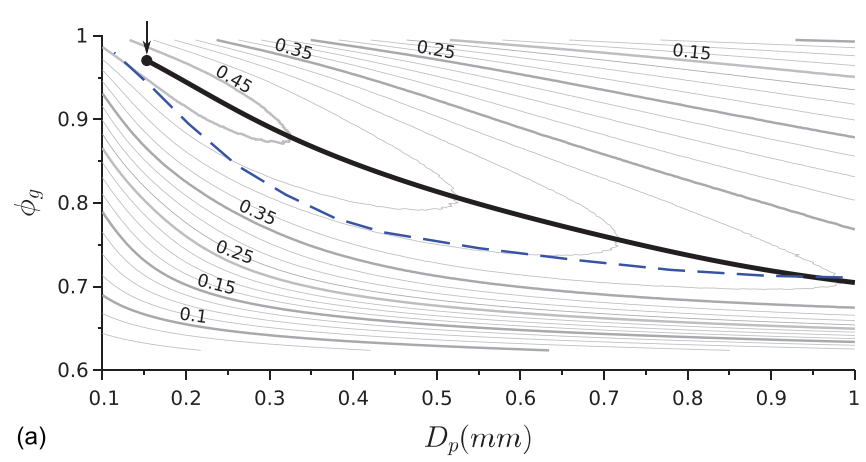

(a)

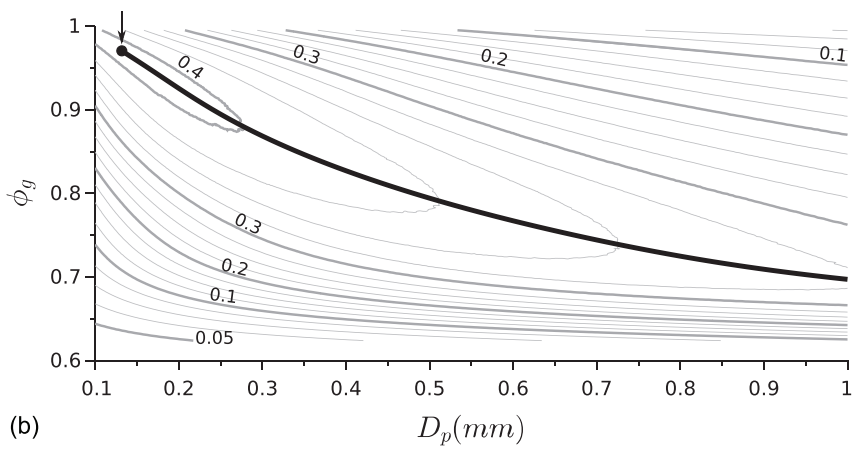

FIG. 13. (Color online) 2D maps of mean sound absorption coefficient $S A A$ as a function of pore size $D_{p}$ and porosity $\phi_{g}=1-\phi_{s}$ : (a) Sample thickness, $25 \mathrm{~mm}$; (b) Sample thickness, $20 \mathrm{~mm}$. The black thick line shows the morphological configurations $\left(D_{p}, \phi_{g}\right)$ maximizing the mean sound absorption coefficient $S A A$. The arrow indicates the best morphological configuration. Gray lines correspond to $S A A$ contour lines. The difference of sound absorption ratings between adjacent contour lines is 0.025 . Numbers indicate to the values of $S A A$ of the thickest contour lines. In (a), the dashed line corresponds to the results obtained by Chevillotte and Perrot (2017) with bcc structure of interpenetrating spheres. predicts SAC values slightly lower than those measured. This small discrepancy could be attributed to a systematic error of the JCAL model to predict the intermediate frequency regime (Cortis et al., 2003; Pride et al., 1993). By using the JCAL model and the approximate formulas provided in Sec. III [Eq. (16)] for visco-thermal parameters with $\phi^{\star}=0.38$, we have calculated $S A A$ for various porosities $\left(\phi_{g}=1-\phi_{s}\right)$ and pore sizes, and solved the optimization problem to maximize $S A A$ for two sample thicknesses. Results are gathered in Fig. 13. As observed with measurements on real foam samples, the optimal configurations of $\left(\phi_{s}, D_{p}\right)$ are located in a range of high porosity $\left(\phi_{s}<0.1\right)$ and small pore sizes $\left(D_{p}<0.3 \mathrm{~mm}\right)$. These results are in qualitative agreement with previous results obtained by Chevillotte and Perrot (2017) with FEM calculations performed on idealized bcc interpenetrating spheres.

\section{CONCLUSION}

This paper presents an experimental and numerical parametric study of the acoustical properties of monodisperse foam. Our numerical model combines the semiphenomenological JCAL model and FEM calculations of visco-thermal parameters performed on bcc foam structure, calculated with SE (surface area minimization software).

A summary of the main results is provided as follows:

- Visco-thermal parameters calculated by FEM on SE bcc foam PUC are similar to the ones measured on real monodisperse foam samples, except for high solid volume fraction $\left(\phi_{s}>0.25\right)$.

- The static viscous permeability $k_{0}$ and the tortuosity $\alpha_{\infty}$ calculated by our numerical simulations compare fairly well with the previously published predictions of effective medium models (Langlois et al., 2018, 2019).

- Approximate formulas based on our numerical results are proposed and can be used to estimate the visco-thermal parameters of real monodisperse foam samples.

- As observed on real foam samples, the optimal configurations of $\left(\phi_{s}, D_{p}\right)$ for normal incidence sound absorption, predicted by the combination of the JCAL model and our approximate formulas, correspond to a range of high porosity $\left(\phi_{s}<0.1\right)$ and small pore sizes $\left(D_{p}<0.3 \mathrm{~mm}\right)$ for a moderate sample thickness $(20-25 \mathrm{~mm})$.

\section{APPENDIX A: JCAL MODEL}

In this appendix, we recall the expressions of the dynamic density $\rho_{e q}$ and bulk modulus $K_{e q}$ of the equivalent fluid medium as provided by the JCAL model (Champoux and Allard, 1991; Johnson et al., 1987; Lafarge et al., 1997) and expressed as in Olny and Panneton (2008; Panneton and Olny, 2006):

$$
\rho_{e q}=\left(\frac{\rho_{0} \alpha_{\infty}}{\phi_{g}}+\frac{\mu}{k_{0} \omega} G_{I}\right)-j \frac{\mu}{k_{0} \omega} G_{R}
$$




$$
\frac{\gamma P_{0}}{K_{e q}}=\gamma-(\gamma-1)\left(1-j \frac{\omega_{t}}{\omega}\left(1+j \frac{M^{\prime}}{2} \frac{\omega}{\omega_{t}}\right)^{1 / 2}\right)^{-1}
$$

where $G_{R}=\left[\frac{1}{2}+\frac{1}{2}\left(1+\left(\frac{M}{2} \frac{\omega}{\omega_{\mu}}\right)^{2}\right)^{1 / 2}\right]^{1 / 2}$ and $G_{I}=\left(M / 4 G_{R}\right)$ $\left(\omega / \omega_{\mu}\right)$, with $M=8 \alpha_{\infty} k_{0} / \phi_{g} \Lambda^{2}, \quad \omega_{\mu}=\mu \phi_{g} / k_{0} \alpha_{\infty} \rho_{0}$, $M^{\prime}=8 k_{0}^{\prime} / \phi_{g} \Lambda^{\prime 2}$, and $\omega_{t}=\mu \phi_{g} / k_{0}^{\prime} P_{r} \rho_{0}$.

In these equations, $P_{0}$ and $\rho_{0}$ are the pressure and mass density, respectively, of the saturating fluid at rest, $\mu$ is its dynamic viscosity, $N_{P r}$ is the Prandtl number $(\approx 0.71$ for air), $\gamma=C_{p} / C_{v}$ is the ratio of the heat capacities at constant pressure and volume, and $j$ the imaginary unit.

The wave number $k_{c}(\omega)$ and the characteristic impedance $Z_{c}(\omega)$ of the equivalent-fluid are given by

$$
k_{c}=\omega\left(\rho_{e q} / K_{e q}\right)^{1 / 2} \text { and } Z_{c}=\left(\rho_{e q} K_{e q}\right)^{1 / 2} .
$$

The sound absorbing coefficient at normal incidence $S A C_{N I}$ of a layer of equivalent-fluid backed by an impervious rigid wall is related to the specific surface impedance $Z_{s}$ of the sample of thickness $H_{s p}$ :

$$
S A C_{N I}=1-\left|\frac{Z_{s}\left(\omega, H_{s p}\right)-Z_{0}}{Z_{s}\left(\omega, H_{s p}\right)+Z_{0}}\right|^{2},
$$

where $Z_{0}=\left(\gamma \rho_{0} P_{0}\right)^{1 / 2}$ is the characteristic impedance of saturating fluid and $Z_{s}\left(\omega, H_{s p}\right)=-j\left(Z_{c}(\omega) / \phi_{g}\right)$ $\cot \left(k_{c}(\omega) H_{s p}\right)$.

\section{APPENDIX B: THERMAL PERMEABILITY OF FIBROUS MATERIALS}

In this appendix, we present the calculation of the thermal permeability for the case of a fibrous material made of
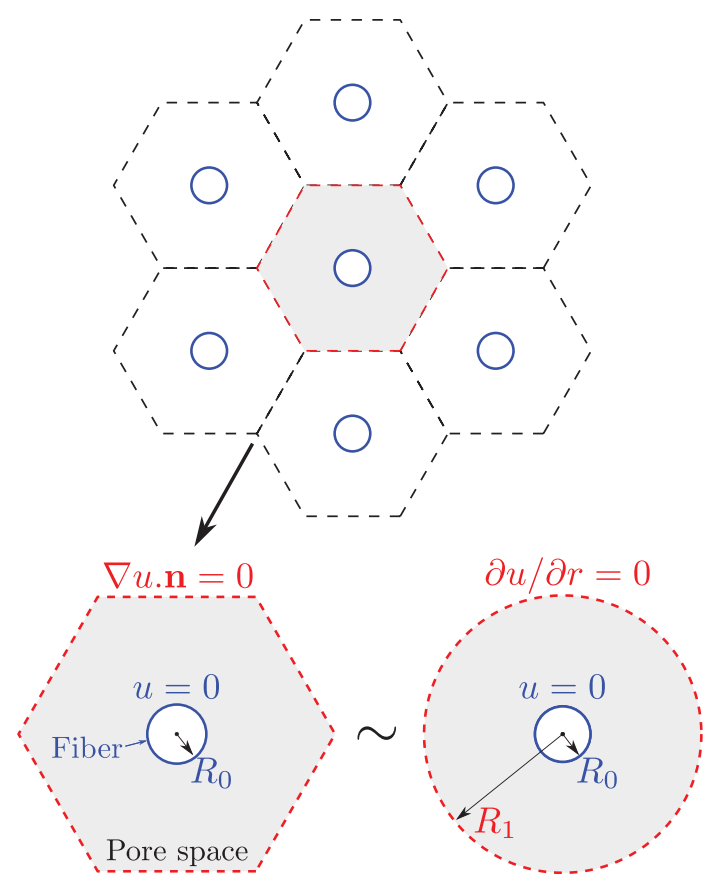

FIG. 14. (Color online) Fiber surrounded by a pore volume. long and straight cylindrical fibers. To simplify the resolution of the problem, the pore volume surrounding the fiber is supposed cylindrical with a "zero flux" boundary condition (Fig. 14). The solid volume fraction is given by $\phi_{s}=R_{0}^{2} /$ $R_{1}^{2}$, and the radius of the pore cylinder $R_{1}$ is linked to the total length of the fiber per unit volume of fibrous material, $\mathcal{L}: R_{1}^{2}=1 / \pi \mathcal{L}$.

The solution of Eq. (10) can be found easily: $\theta(r)$ $=\left(R_{1}^{2} / 2\right) \ln \left(r / R_{0}\right)+\frac{1}{4}\left(R_{0}^{2}-r^{2}\right)$. By averaging this solution over the pore volume, we find the thermal permeability $k_{0}^{\prime}$ :

$$
k_{0}^{\prime}=\frac{1}{8 \pi \mathcal{L}}\left[-2 \ln \left(\phi_{s}\right)-3+4 \phi_{s}+\phi_{s}^{2}\right] .
$$

In the limit of low solid volume fraction, the structure of solid foam samples is quite similar to fibrous material. If we consider a BCC foam sample made of 24 ligaments per PUC, having a length equal to $D_{t} / 2^{3 / 2}$, we can calculate the total length of the fiber per unit volume: $\mathcal{L}=12 / 2^{1 / 2} D_{t}^{2}$. The thermal permeability is then given by

$$
k_{0}^{\prime} \approx \frac{2^{1 / 2} D_{t}^{2}}{96 \pi}\left[-2 \ln \left(\phi_{s}\right)-3+4 \phi_{s}+\phi_{s}^{2}\right] .
$$

The approximate formula for $k_{0}^{\prime}$ given in Eq. (16) is based on Eq. (B2). However, coefficients inside the brackets are adjusted to have a better fit data.

\section{APPENDIX C: THERMAL PERMEABILITY OF SPHERICAL PORES}

In this appendix, we present the calculation of the thermal permeability for the limit case of a spherical pore. We consider that the solution of Eq. (10) has a spherical symmetry $\theta(r)$, where $r$ is the distance to the pore center. With straightforward steps, we solve Eq. (10): $\theta(r)=\left(R_{p}^{2}-r^{2}\right) / 6$. By averaging this solution over the pore volume, we find the thermal permeability $k_{0}^{\prime} / D_{p}^{2}=\left(1-\phi_{s}\right) / 60$.

${ }^{1}$ See supplementary material at https://doi.org/10.1121/10.0001995 for comparison between measurements and JCAL model predictions of dynamic density $\rho_{\text {eq }}$ and bulk modulus $\mathrm{K}_{\text {eq. }}$.

Allard, J. F., and Atalla, N. (2009). Propagation of Sound in Porous Media (John Wiley and Sons, West Sussex, UK).

ASTM C423-17. (2017). "Standard Test Method for Sound Absorption and Sound Absorption Coefficients by the Reverberation Room Method" (ASTM International, West Conshohocken, PA).

Brakke, K. A. (1992). "The surface evolver," Exp. Math. 1(2), 141-165.

Brown, R. J. S. (1980). "Connection between formation factor for electrical resistivity and fluid-solid coupling factor in Biot's equations for acoustic waves in fluid-filled porous media," Geophysics 45(8), 1269-1275.

Cantat, I., Cohen-Addad, S., Elias, F., Graner, F., Höhler, R., Pitois, O., Rouyer, F., and Saint-Jalmes, A. (2013). Foams: Structure and Dynamics (Oxford University Press, Oxford).

Champoux, Y., and Allard, J. F. (1991). "Dynamic tortuosity and bulk modulus in air-saturated porous media," J. Appl. Phys. 70(4), 1975-1979.

Chevillotte, F., and Perrot, C. (2017). "Effect of the three-dimensional microstructure on the sound absorption of foams: A parametric study," J. Acoust. Soc. Am. 142(2), 1130-1140.

Chevillotte, F., Perrot, C., and Guillon, E. (2013). "A direct link between microstructure and acoustical macro-behavior of real double porosity foams," J. Acoust. Soc. Am. 134(6), 4681-4690. 
Cortis, A., Smeulders, D. M. J., Guermond, J. L., and Lafarge, D. (2003). "Influence of pore roughness on high-frequency permeability," Phys. Fluids 15(6), 1766-1775.

Despois, J. F., and Mortensen, A. (2005). "Permeability of open-pore microcellular materials," Acta Mater. 53(5), 1381-1388.

Doutres, O., Atalla, N., and Dong, K. (2011). "Effect of the microstructure closed pore content on the acoustic behavior of polyurethane foams," J. Appl. Phys. 110(6), 064901.

Doutres, O., Atalla, N., and Dong, K. (2013). "A semi-phenomenological model to predict the acoustic behavior of fully and partially reticulated polyurethane foams," J. Appl. Phys. 113(5), 054901.

Doutres, O., Salissou, Y., Atalla, N., and Panneton, R. (2010). "Evaluation of the acoustic and non-acoustic properties of sound absorbing materials using a three-microphone impedance tube," Appl. Acoust. 71(6), 506-509.

Drenckhan, W., and Hutzler, S. (2015). "Structure and energy of liquid foams," Adv. Colloid Interface Sci. 224, 1-16.

Feneuil, B., Aimedieu, P., Scheel, M., Perrin, J., Roussel, N., and Pitois, O. (2019). "Stability criterion for fresh cement foams," Cem. Concr. Res. 125, 105865.

Firdaouss, M., Guermond, J.-L., and Lafarge, D. (1998). "Some remarks on the acoustic parameters of sharp-edged porous media," Int. J. Eng. Sci. 36(9), 1035-1046.

Gandolfo, F. G., and Rosano, H. L. (1997). "Interbubble gas diffusion and the stability of foams," J. Colloid Interface Sci. 194(1), 31-36.

Gibson, L. J., and Ashby, M. F. (1997). Cellular Solids (Cambridge University Press, Cambridge), available at https://www.cambridge.org/ core/product/identifier/9781139878326/type/book.

Hall, J. E. (1975). "Access resistance of a small circular pore," J. Gen. Physiol. 66, 531-532.

Henry, M., and Allard, J. F. (1997). "Acoustical measurement of the trapping constant of foams with open cells," Comptes Rendus l'Academie Sci. - Ser. IIb Mec. Phys. Chim. Astron. 325, 331-338.

Hoang, M. T., and Perrot, C. (2012). "Solid films and transports in cellular foams," J. Appl. Phys. 112(5), 054911.

Hung, T.-C., Huang, J.-S., Wang, Y.-W., and Lin, K.-Y. (2014). "Inorganic polymeric foam as a sound absorbing and insulating material," Constr. Build. Mater. 50, 328-334.

Jang, W.-Y., Kraynik, A. M., and Kyriakides, S. (2008). "On the microstructure of open-cell foams and its effect on elastic properties," Int. J. Solids Struct. 45(7-8), 1845-1875.

Johnson, D. L., Koplik, J., and Dashen, R. (1987). "Theory of dynamic permeability and tortuosity in fluid saturated porous media," J. Fluid Mech. 176, 379-402.

Kaddami, A., and Pitois, O. (2019). "A physical approach towards controlling the microstructure of metakaolin-based geopolymer foams," Cem. Concr. Res. 124, 105807.
Kirkpatrick, S. (1973). "Percolation and conduction," Rev. Mod. Phys. 45, 574-588.

Kraynik, A. M., Reinelt, D. A., and van Swol, F. (2003). "Structure of random monodisperse foam,” Phys. Rev. E 67(3), 031403.

Kraynik, A. M., Reinelt, D. A., and van Swol, F. (2004). "Structure of random foam,” Phys. Rev. Lett. 93(20), 208301.

Kumar, P., and Topin, F. (2014). "Micro-structural impact of different strut shapes and porosity on hydraulic properties of Kelvin-like metal foams," Transp. Porous Media 105(1), 57-81.

Lafarge, D., Lemarinier, P., Allard, J. F., and Tarnow, V. (1997). "Dynamic compressibility of air in porous structures at audible frequencies," J. Acoust. Soc. Am. 102(4), 1995-2006.

Langlois, V., Trinh, V. H., Lusso, C., Perrot, C., Chateau, X., Khidas, Y., and Pitois, O. (2018). "Permeability of solid foam: Effect of pore connections," Phys. Rev. E 97(5), 053111.

Langlois, V., Trinh, V. H., and Perrot, C. (2019). "Electrical conductivity and tortuosity of solid foam: Effect of pore connections," Phys. Rev. E 100(1), 013115.

Murtagh, R., Whyte, D., Weaire, D., and Hutzler, S. (2015). "Adaptation of the Z-cone model to the estimation of the energy of a bcc foam," Philos. Mag. 95(35), 4023-4034.

Olny, X., and Panneton, R. (2008). "Acoustical determination of the parameters governing thermal dissipation in porous media," J. Acoust. Soc. Am. 123(2), 814-824.

Panneton, R., and Olny, X. (2006). "Acoustical determination of the parameters governing viscous dissipation in porous media," J. Acoust. Soc. Am. 119(4), 2027-2040.

Perrot, C., Chevillotte, F., Hoang, M. T., Bonnet, G., Bécot, F. X., Gautron, L., and Duval, A. (2012). "Microstructure, transport, and acoustic properties of open-cell foam samples: Experiments and three-dimensional numerical simulations," J. Appl. Phys. 111(1), 014911.

Pitois, O., Kaddami, A., and Langlois, V. (2020). "Capillary imbibition in open-cell monodisperse foams," J. Colloid Interface Sci. 571, 166-173.

Pitois, O., Lorenceau, E., Louvet, N., and Rouyer, F. (2009). "Specific surface area model for foam permeability," Langmuir 25(1), 97-100.

Pride, S. R., Morgan, F. D., and Gangi, A. F. (1993). "Drag forces of porous-medium acoustics," Phys. Rev. B 47(9), 4964-4978.

Sampson, R. A. (1891). "On Stokes's current function," Philo. Trans. R. Soc. London A 182, 449-518.

Trinh, V. H., Langlois, V., Guilleminot, J., Perrot, C., Khidas, Y., and Pitois, O. (2019). "Tuning membrane content of sound absorbing cellular foams: Fabrication, experimental evidence and multiscale numerical simulations," Mater. Des. 162, 345-361.

Zhang, Z., Provis, J. L., Reid, A., and Wang, H. (2014). "Geopolymer foam concrete: An emerging material for sustainable construction," Constr. Build. Mater. 56, 113-127. 